\title{
Convergence analysis of generalized viscosity implicit rules for a nonexpansive semigroup with gauge functions
}

\author{
Pongsakorn Sunthrayuth ${ }^{\mathrm{a}}$, Nuttapol Pakkaranang ${ }^{\mathrm{b}}$, Poom Kumam $^{\mathrm{b}, \mathrm{c}, \mathrm{d}, *}$ \\ ${ }^{a}$ Department of Mathematics and Computer Science, Faculty of Science and Technology, Rajamangala University of Technology \\ Thanyaburi (RMUTT), Thanyaburi, Pathumthani, 12110, Thailand. \\ ${ }^{b}$ KMUTTFixed Point Research Laboratory, Department of Mathematics, Room SCL 802 Fixed Point Laboratory, Science Laboratory \\ Building, Faculty of Science, King Mongkut's University of Technology Thonburi (KMUTT), 126 Pracha-Uthit Road, Bang Mod, Thung \\ Khru, Bangkok 10140, Thailand. \\ ${ }^{c}$ KMUTT-Fixed Point Theory and Applications Research Group, Theoretical and Computational Science Center (TaCS), Science \\ Laboratory Building, Facuty of Science, King Mongkut's University of Technology Thonburi (KMUTT), 126 Pracha-Uthit Road, Bang \\ Mod, Thung Khru, Bangkok 10140, Thailand. \\ ${ }^{d}$ Department of Medical Research, China Medical University Hospital, China Medical University, Taichung 40402, Taiwan.
}

Communicated by Q. Dong

\begin{abstract}
In this paper, we introduce an iterative algorithm for finding the set of common fixed points of nonexpansive semigroups by the generalized viscosity implicit rule in certain Banach spaces which has a uniformly Gâteaux differentiable norm and admits the duality mapping $j_{\varphi}$, where $\varphi$ is a gauge function. We prove strong convergence theorems of proposed algorithm under appropriate conditions. As applications, we apply main result to solving the fixed point problems of countable family of nonexpansive mappings and the problems of zeros of accretive operators. Furthermore, we give some numerical examples for supporting our main results.
\end{abstract}

Keywords: Nonexpansive semigroup, Banach spaces, strong convergence, fixed point problem, iterative method. 2010 MSC: 47H09, 47H10, 47J05, 47J25.

(C)2018 All rights reserved.

\section{Introduction}

In this paper, we assume that $E$ is a real Banach space with dual space $E^{*}$ and $C$ is a nonempty subset of $E$. Let $T: C \rightarrow C$ be a mapping. We denote the set of all fixed points of $T$ by $F(T)=\{x \in C: x=T x\}$. A mapping $\mathrm{T}: \mathrm{C} \rightarrow \mathrm{C}$ is called nonexpansive if for each $\mathrm{x}, \mathrm{y} \in \mathrm{C}$ such that

$$
\|T x-T y\| \leqslant\|x-y\| .
$$

\footnotetext{
*Corresponding author

Email addresses: pongsakorn_su@rmutt.ac.th (Pongsakorn Sunthrayuth), nuttapol.pak@mail.kmutt.ac.th (Nuttapol Pakkaranang), poom.kum@kmutt.ac.th (Poom Kumam)
}

doi: 10.22436/jnsa.011.09.02

Received: 2017-12-08 Revised: 2018-04-01 Accepted: 2018-04-05 
A mapping $f: C \rightarrow C$ is called a contraction, if there exists a constant $\rho \in(0,1)$ and for each $x, y \in C$

$$
\|f(x)-f(y)\| \leqslant \rho\|x-y\| .
$$

The viscosity approximation method has been successfully applied to various problems from calculus of variations as minimal surface problems and plasticity theory and phase transition. Various applications can be obtained in optimal control theory, singular perturbations, game theory, and partial differential equations (see [4] and references therein). In recent years, viscosity approximation method for approximating the set of (common) fixed points of nonlinear mappings have been investigated extensively by many authors in Hilbert and Banach spaces (see [10, 11, 13, 19, 20, 23-25, 30] and the references therein).

Very recently, the implicit midpoint rule (IMR) has become a powerful numerical method for numerically solving ordinary differential equations (in particular, the stiff equations) (see $[5,6,14,21,22,28]$ ) and differential algebraic equations (see [32]).

Xu et al. [31] combined the Moudafi's viscosity method [19] (see also [30]) with IMR for nonexpansive mappings $\mathrm{T}$ and proposed the following viscosity implicit midpoint rule (VIMR) in Hilbert spaces $\mathrm{H}$ as follows:

$$
x_{n+1}=\alpha_{n} f\left(x_{n}\right)+\left(1-\alpha_{n}\right) T\left(\frac{x_{n}+x_{n+1}}{2}\right), \forall n \geqslant 1,
$$

where $\left\{\alpha_{n}\right\}$ is a real control condition in $(0,1)$. They also proved that VIMR converges strongly to a point $x^{*} \in \mathrm{F}(\mathrm{T})$ which also solves the variational inequality

$$
\left\langle(\mathrm{f}-\mathrm{I}) x^{*}, z-x^{*}\right\rangle \leqslant 0, \quad \forall z \in \mathrm{F}(\mathrm{T}),
$$

where I is the identity on $\mathrm{H}$.

Later, Ke and Ma [17] improved the VIMR (1.1) by replacing the midpoint by any point of interval $\left[x_{n}, x_{n+1}\right]$. They introduced the so-call generalized viscosity implicit midpoint rules to approximating the fixed point of nonexpansive mapping $\mathrm{T}$ in Hilbert spaces $\mathrm{H}$. They obtained the following result.

Theorem 1.1 (Theorem KM). Let $\mathrm{C}$ be a nonempty, closed, and convex subset of a real Hilbert space $\mathrm{H}$. Let $\mathrm{T}: \mathrm{C} \rightarrow \mathrm{C}$ be a nonexpansive mapping with $\mathrm{F}(\mathrm{T}) \neq \emptyset$ and let $\mathrm{f}$ be a contraction on $\mathrm{C}$ with coefficient $\rho \in(0,1)$. Let $\mathrm{x}_{1} \in \mathrm{C}$, and $\left\{\mathrm{x}_{\mathrm{n}}\right\}$ be a sequence generated by

$$
x_{n+1}=\alpha_{n} f\left(x_{n}\right)+\beta_{n} x_{n}+\gamma_{n} T\left(s_{n} x_{n}+\left(1-s_{n}\right) x_{n+1}\right), \quad \forall n \geqslant 1,
$$

where $\left\{\alpha_{n}\right\},\left\{\beta_{n}\right\},\left\{\gamma_{n}\right\}$, and $\left\{s_{n}\right\}$ are sequences in $(0,1)$ with $\alpha_{n}+\beta_{n}+\gamma_{n}=1$. Suppose that the following conditions hold:

(C1) $\lim _{n \rightarrow \infty} \gamma_{n}=1$;

(C2) $\sum_{n=1}^{\infty} \alpha_{n}=\infty$ and $\sum_{n=1}^{\infty}\left|\alpha_{n+1}-\alpha_{n}\right|<\infty$;

(C3) $\sum_{n=1}^{\infty}\left|\beta_{n+1}-\beta_{n}\right|<\infty$;

(C4) $0<\mathrm{k} \leqslant \mathrm{s}_{\mathrm{n}} \leqslant \mathrm{s}_{\mathrm{n}+1}<1$ for all $\mathrm{n} \geqslant 1$.

Then $\left\{x_{n}\right\}$ converges strongly to a point $x^{*} \in \mathrm{F}(\mathrm{T})$, which also solves (1.2).

The above results naturally bring us to the following questions.

Question 1: Can we obtain strong convergence result of Theorem 1.1 to higher spaces other than Hilbert spaces? Such as a real reflexive strictly convex Banach space which has a uniformly Gâteaux differentiable norm and admits the duality mapping $j_{\varphi}$, where $\varphi$ is a gauge function.

Question 2: Can we remove the control condition (C1) in Theorem 1.1?

Question 3: Can we weaken the control conditions (C2) and (C3) in Theorem 1.1?

Question 4: Can we extend the generalized viscosity implicit midpoint rules (1.3) to finding the set of common fixed points of a family of mappings? Such as one-parameter semigroups of nonexpansive mappings. 
The main objective in this paper is to give an affirmative answer to above questions, we introduce an iterative algorithm for finding the set of common fixed points of nonexpansive semigroups by the generalized viscosity implicit rule in a real reflexive strictly convex Banach space which has a uniformly Gâteaux differentiable norm and admits the duality mapping $j_{\varphi}$, where $\varphi$ is a gauge function. Then, we prove strong convergence theorems of proposed algorithm with different approach on control conditions. As applications, we apply main results to solving the fixed point problems of family of nonexpansive mappings and the problems of zeros of accretive operators. Furthermore, we also give some numerical examples for support our main results.

\section{Preliminaries}

The continuous and strictly increasing function $\varphi:[0, \infty) \rightarrow[0, \infty)$ is said to be gauge function if $\varphi(0)=0$ and $\varphi(t) \rightarrow \infty$ as $\mathrm{t} \rightarrow \infty$. The duality mapping $\mathrm{J}_{\varphi}: \mathrm{E} \rightarrow 2^{\mathrm{E}^{*}}$ associated with a gauge function $\varphi$ is defined by

$$
\mathrm{J}_{\varphi}(x)=\left\{\mathrm{f}^{*} \in \mathrm{E}^{*}:\left\langle x, \mathrm{f}^{*}\right\rangle=\|x\| \varphi(\|x\|),\left\|\mathrm{f}^{*}\right\|=\varphi(\|x\|), \quad \forall x \in E\right\},
$$

where $\langle\cdot, \cdot\rangle$ denotes the generalized duality paring. In particular, the duality mapping with the gauge function $\varphi(t)=t$, denoted by J is referred to as the normalized duality mapping. In this case $\varphi(t)=t^{q-1}$, $q>1$, the duality mapping $J_{\varphi}=J_{q}$ is called generalized duality mapping. It follows from the definition that $\mathrm{J}_{\varphi}(\mathrm{x})=\frac{\varphi(\|x\|)}{\|x\|} \mathrm{J}(\mathrm{x})$ for each $\mathrm{x} \neq 0$, and $\mathrm{J}_{\mathrm{q}}(\mathrm{x})=\|\mathrm{x}\|^{\mathrm{q}-2} \mathrm{~J}(\mathrm{x}), \mathrm{q}>1$ (see [9]).

Remark 2.1. For the gauge function $\varphi$, the function $\Phi:[0, \infty) \rightarrow[0, \infty)$ defined by $\Phi(t)=\int_{0}^{t} \varphi(\tau) d \tau$ is continuous, convex, and strictly increasing function on $[0, \infty)$. Therefore, $\Phi$ has a continuous inverse function $\Phi^{-1}$.

Remark 2.2. It is observe that if $k \in[0,1]$ then $\varphi(k y) \leqslant \varphi(y)$. Then, we have

$$
\Phi(k t)=\int_{0}^{k t} \varphi(\tau) d \tau=k \int_{0}^{t} \varphi(k y) d y \leqslant k \int_{0}^{t} \varphi(y) d y=k \Phi(t) .
$$

Remark 2.3. If a Banach space $\mathrm{E}$ has a uniformly Gâteaux differentiable, then $\mathrm{J} \varphi$ is single-valued and also denoted by $j_{\varphi}$.

Lemma 2.4 ([18]). Let $\mathrm{E}$ be a Banach space. Then for each $\mathrm{x}, \mathrm{y} \in \mathrm{E}$, the following inequality holds:

$$
\Phi(\|x+y\|) \leqslant \Phi(\|x\|)+\left\langle y, j_{\varphi}(x+y)\right\rangle, j_{\varphi}(x+y) \in J_{\varphi}(x+y) .
$$

Definition 2.5. A one-parameter family $\mathcal{S}=\left\{\mathrm{T}_{\mathrm{t}}\right\}_{\mathrm{t} \geqslant 0}: \mathrm{C} \rightarrow \mathrm{C}$ is said to be a nonexpansive semigroup if it satisfies the following conditions:

(S1) $T_{0} x=x$ for $x \in C$;

(S2) $T_{s+t}=T_{s} T_{t}$ for $s, t \geqslant 0$;

(S3) $\lim _{\mathrm{t} \rightarrow 0^{+}} \mathrm{T}(\mathrm{t}) \mathrm{x}=\mathrm{x}$ for $\mathrm{x} \in \mathrm{C}$;

(S4) for each $t \geqslant 0, T_{t}$ is nonexpansive, i.e.,

$$
\left\|T_{t} x-T_{t} y\right\| \leqslant\|x-y\|, \forall x, y \in C .
$$

Remark 2.6. We denote by $F(\mathcal{S})$ the set of all common fixed points of $\mathcal{S}$, i.e., $F(\mathcal{S})=\bigcap_{t \geqslant 0} F\left(T_{t}\right)$.

Now, we give some examples of semigroup operator. The following classical examples were the main sources for the development of semigroup theory (see [15]). 
Example 2.7. Let $E$ be a real Banach space and let $\mathcal{L}(E)$ be the space of all bounded linear operators on $E$. For $A \in \mathcal{L}(E)$, consider the initial value problem for a linear autonomous differential equation on $[0, \infty)$ :

$$
u^{\prime}(t)=A u(t), u(0)=x .
$$

Notice that the solution of problem (2.1) is given by

$$
u(t):=T_{t} x \text { for all } t \geqslant 0 .
$$

Then, we can show that the operator $T_{t} x$ is a semigroup on $E$.

Example 2.8. Let $E:=L^{p}\left(\mathbb{R}^{n}\right), 1 \leqslant p<\infty$. Consider the initial value problem for the heat equation:

$$
\begin{aligned}
& \frac{\partial u}{\partial t}=\Delta u, \quad \text { for } x \in \mathbb{R}^{n} \text { and } t>0, \\
& u(x, 0)=f(x), \text { for } x \in \mathbb{R}^{n},
\end{aligned}
$$

where $\Delta=\sum_{i=1}^{n} \frac{\partial^{2}}{\partial x_{i}^{2}}$ is the Laplacian operator on E. By using Fourier transform, we can write the solution $u(x, t)$ in the form of convolution integral as:

$$
u(x, t)=\frac{1}{\sqrt{(4 \pi t)^{n}}} \int_{\mathbb{R}^{n}} e^{\frac{-\|x-\xi\|^{2}}{4 t}} f(\xi) d \xi=\left(K_{t} * f\right)(x),
$$

where $t>0, f \in E$, and $K_{t}$ is the heat kernel given by $K_{t}(x)=\frac{1}{\sqrt{(4 \pi t)^{n}}} e^{\frac{-\|x\|^{2}}{4 t}}$. Then the solution of problem (2.2) can be written as:

$$
T_{t} f(x):=u(x, t)=\left(K_{t} * f\right)(x) .
$$

We can show that the operator $T_{t} f(x)$ is a semigroup on $E$.

Definition $2.9([1,2,8])$. A continuous operator semigroup $\mathcal{S}=\left\{T_{t}\right\}_{t} \geqslant 0: C \rightarrow C$ is said to be uniformly asymptotically regular (in short, u.a.r.) if for all $s \geqslant 0$ and any bounded subset $B$ of $C$,

$$
\lim _{t \rightarrow \infty} \sup _{x \in B}\left\|T_{t} x-T_{s} T_{t} x\right\|=0 .
$$

Example 2.10. Let $C$ be a closed convex subset of a uniformly convex Banach space $E$. Let $\mathcal{S}=\left\{T_{t}\right\}_{t} \geqslant 0$ : $C \rightarrow C$ be a nonexpansive semigroup. Let $\left\{\sigma_{t}\right\}_{t>0}$ defined by $\sigma_{t} x=\frac{1}{t} \int_{0}^{t} T_{s} x d s$. Then, for each $h>0$ and any bounded subset $B$ of $C$, we have

$$
\left\|\sigma_{t} x-\sigma_{h} \sigma_{t} x\right\|=\left\|\sigma_{t} x-\frac{1}{h} \int_{0}^{h} T_{s} \sigma_{t} x d s\right\|=\left\|\frac{1}{h} \int_{0}^{h}\left(\sigma_{t} x-T_{s} \sigma_{t} x\right) d s\right\| \leqslant \frac{1}{h} \int_{0}^{h}\left\|\sigma_{t} x-T_{s} \sigma_{t} x\right\| d s .
$$

From Lemma 2.7 of [12], we have

$$
\lim _{t \rightarrow \infty} \sup _{x \in B}\left\|\sigma_{t} x-\sigma_{h} \sigma_{t} x\right\| \leqslant \frac{1}{h} \int_{0}^{h} \lim _{t \rightarrow \infty} \sup _{x \in B}\left\|\sigma_{t} x-T_{s} \sigma_{t} x\right\| d s=0,
$$

i.e., $\left\{\sigma_{t}\right\}_{t}>0$ is u.a.r..

Theorem 2.11 ([13]). Let $\mathrm{C}$ be a nonempty, closed, and convex subset of a real reflexive strictly convex Banach space $\mathrm{E}$, which has a uniformly Gâteaux differentiable norm and admits the duality mapping $j_{\varphi}$. Let $\mathcal{S}=\left\{\mathrm{T}_{\mathrm{t}}\right\}_{\mathrm{t} \geqslant 0}: \mathrm{C} \rightarrow \mathrm{C}$ be a u.a.r. nonexpansive semigroup such that $\mathrm{F}(\mathcal{S}):=\bigcap_{\mathrm{t} \geqslant 0} \mathrm{~F}\left(\mathrm{~T}_{\mathrm{t}}\right) \neq \emptyset$ and $\mathrm{f}$ be a contraction on $\mathrm{C}$ with coefficient $\rho \in(0,1)$. Suppose that $\left\{t_{n}\right\}$ is a real divergent sequence and $\left\{\alpha_{n}\right\}$ is a real sequence in $(0,1)$ with $\lim _{n \rightarrow \infty} \alpha_{n}=0$. Then, the sequence $\left\{x_{n}\right\}$ defined by

$$
x_{n}=\alpha_{n} f\left(x_{n}\right)+\left(1-\alpha_{n}\right) T_{t_{n}} x_{n}, \forall n \geqslant 1,
$$

converges strongly to a point $p \in \mathrm{F}(\mathcal{S})$, which also solves the variational inequality

$$
\left\langle f(p)-p, j_{\varphi}(z-p)\right\rangle \leqslant 0, \quad \forall z \in F(\mathcal{S}) .
$$


Lemma 2.12 ([26]). Let $\left\{x_{n}\right\}$ and $\left\{l_{n}\right\}$ be bounded sequences in a Banach space $E$ and let $\left\{\beta_{n}\right\}$ be a sequence in $[0,1]$ with $0<\liminf _{n \rightarrow \infty} \beta_{n} \leqslant \limsup _{n \rightarrow \infty} \beta_{n}<1$. Suppose $x_{n+1}=\left(1-\beta_{n}\right) l_{n}+\beta_{n} x_{n}$ for all integers $n \geqslant 0$ and $\lim \sup _{n \rightarrow \infty}\left(\left\|l_{n+1}-l_{n}\right\|-\left\|x_{n+1}-x_{n}\right\|\right) \leqslant 0$. Then, $\lim _{n \rightarrow \infty}\left\|l_{n}-x_{n}\right\|=0$.

Lemma 2.13 ([29]). Assume that $\left\{a_{n}\right\}$ is a nonnegative real sequence such that

$$
a_{n+1} \leqslant\left(1-\theta_{n}\right) a_{n}+\theta_{n} \sigma_{n}
$$

where $\left\{\theta_{n}\right\}$ is a sequence in $(0,1)$ and $\left\{\sigma_{n}\right\}$ is a real sequence such that

(i) $\sum_{n=1}^{\infty} \theta_{n}=\infty$;

(ii) $\limsup _{n \rightarrow \infty} \sigma_{n} \leqslant 0$ or $\sum_{n=1}^{\infty}\left|\theta_{n} \sigma_{n}\right|<\infty$.

Then, $\lim _{n \rightarrow \infty} a_{n}=0$.

\section{Main results}

Theorem 3.1. Let $\mathrm{C}$ be a nonempty, closed, and convex subset of a real reflexive strictly convex Banach space $\mathrm{E}$, which has a uniformly Gâteaux differentiable norm and admits the duality mapping $j_{\varphi}$. Let $\mathcal{S}=\left\{\mathrm{T}_{\mathrm{t}}\right\}_{\mathrm{t} \geqslant 0}: \mathrm{C} \rightarrow \mathrm{C}$ be a u.a.r. nonexpansive semigroup such that $\mathrm{F}(\mathcal{S}):=\bigcap_{\mathrm{t} \geqslant 0} \mathrm{~F}\left(\mathrm{~T}_{\mathrm{t}}\right) \neq \emptyset$ and $\mathrm{f}$ be a contraction on $\mathrm{C}$ with coefficient $\rho \in(0,1)$. For given $x_{1} \in C$, let $\left\{x_{n}\right\}$ be a sequence generated by

$$
x_{n+1}=\alpha_{n} f\left(x_{n}\right)+\beta_{n} x_{n}+\gamma_{n} T_{t_{n}}\left(s_{n} x_{n}+\left(1-s_{n}\right) x_{n+1}\right), \quad \forall n \geqslant 1,
$$

where $\left\{\alpha_{n}\right\},\left\{\beta_{n}\right\},\left\{\gamma_{n}\right\},\left\{s_{n}\right\} \subset(0,1)$ with $\alpha_{n}+\beta_{n}+\gamma_{n}=1$, and $\left\{t_{n}\right\} \subset(0, \infty)$ satisfying the following conditions:

(C1) $\lim _{n \rightarrow \infty} \alpha_{n}=0$ and $\sum_{n=1}^{\infty} \alpha_{n}=\infty$;

(C2) $\lim _{n \rightarrow \infty}\left|\beta_{n+1}-\beta_{n}\right|=0$ and $0<\liminf _{n \rightarrow \infty} \beta_{n} \leqslant \limsup _{n \rightarrow \infty} \beta_{n}<1$;

(C3) $t_{n+1}=h+t_{n}$ for all $h \geqslant 0$ and $\lim _{n \rightarrow \infty} t_{n}=\infty$;

(C4) $0<\mathrm{k} \leqslant s_{n} \leqslant s_{n+1}<1$ for all $n \geqslant 1$.

Then, $\left\{x_{n}\right\}$ defined by (3.1) converges strongly to a point $p \in F(\mathcal{S})$, which also solves the variational inequality

$$
\left\langle f(p)-p, j_{\varphi}(z-p)\right\rangle \leqslant 0, \forall z \in F(\mathcal{S}) .
$$

Proof. First, we will show that $\left\{x_{n}\right\}$ generated by (3.1) is well defined. For each $x, u \in C$, define the mapping $\mathrm{S}_{\mathrm{n}}: \mathrm{C} \rightarrow \mathrm{C}$ by

$$
S_{n} x:=\alpha_{n} f(x)+\beta_{n} x+\gamma_{n} T_{t_{n}}\left(s_{n} u+\left(1-s_{n}\right) x\right), \quad \forall n \geqslant 1 .
$$

For each $x, y \in C$, we have

$$
\begin{aligned}
\left\|S_{n} x-S_{n} y\right\| & =\left\|\alpha_{n}(f(x)-f(y))+\beta_{n}(x-y)+\gamma_{n}\left[T_{t_{n}}\left(s_{n} u+\left(1-s_{n}\right) x\right)-T_{t_{n}}\left(s_{n} u+\left(1-s_{n}\right) y\right)\right]\right\| \\
& \leqslant \alpha_{n}\|f(x)-f(y)\|+\beta_{n}\|x-y\|+\gamma_{n}\left\|T_{t_{n}}\left(s_{n} u+\left(1-s_{n}\right) x\right)-T_{t_{n}}\left(s_{n} u+\left(1-s_{n}\right) y\right)\right\| \\
& \leqslant \alpha_{n} \rho\|x-y\|+\beta_{n}\|x-y\|+\gamma_{n}\left(1-s_{n}\right)\|x-y\| \\
& =\left(1-(1-\rho) \alpha_{n}-\gamma_{n} \kappa\right)\|x-y\| \leqslant\left(1-(1-\rho) \alpha_{n}\right)\|x-y\|,
\end{aligned}
$$

this mean that $S_{n}$ is a contraction. So $S_{n}$ has a unique fixed point. Therefore, the sequence $\left\{x_{n}\right\}$ defined by (3.1) is well-defined.

Next, we show that $\left\{x_{n}\right\}$ is bounded. For each $p \in F(\mathcal{S})$, we have

$$
\begin{aligned}
\left\|x_{n+1}-p\right\| & =\left\|\alpha_{n}\left(f\left(x_{n}\right)-p\right)+\beta_{n}\left(x_{n}-p\right)+\gamma_{n}\left(T_{t_{n}}\left(s_{n} x_{n}+\left(1-s_{n}\right) x_{n+1}\right)-p\right)\right\| \\
& \leqslant \alpha_{n}\left\|f\left(x_{n}\right)-p\right\|+\beta_{n}\left\|x_{n}-p\right\|+\gamma_{n}\left\|T_{t_{n}}\left(s_{n} x_{n}+\left(1-s_{n}\right) x_{n+1}\right)-p\right\|
\end{aligned}
$$




$$
\begin{aligned}
& \leqslant \alpha_{n}\left\|f\left(x_{n}\right)-p\right\|+\beta_{n}\left\|x_{n}-p\right\|+\gamma_{n}\left\|s_{n}\left(x_{n}-p\right)+\left(1-s_{n}\right)\left(x_{n+1}-p\right)\right\| \\
& \leqslant \alpha_{n}\left\|f\left(x_{n}\right)-f(p)\right\|+\alpha_{n}\|f(p)-p\|+\beta_{n}\left\|x_{n}-p\right\|+r_{n}\left(s_{n}\left\|x_{n}-p\right\|+\left(1-s_{n}\right)\left\|x_{n+1}-p\right\|\right) \\
& \leqslant\left(\alpha_{n} \rho+\beta_{n}+\gamma_{n} s_{n}\right)\left\|x_{n}-p\right\|+\alpha_{n}\|f(p)-p\|+\gamma_{n}\left(1-s_{n}\right)\left\|x_{n+1}-p\right\|,
\end{aligned}
$$

which implies that

$$
\begin{aligned}
\left\|x_{n+1}-p\right\| & \leqslant \frac{\alpha_{n} \rho+\beta_{n}+\gamma_{n} s_{n}}{1-\gamma_{n}\left(1-s_{n}\right)}\left\|x_{n}-p\right\|+\frac{\alpha_{n}}{1-\gamma_{n}\left(1-s_{n}\right)}\|f(p)-p\| \\
& =\left(1-\frac{(1-\rho) \alpha_{n}}{1-\gamma_{n}\left(1-s_{n}\right)}\right)\left\|x_{n}-p\right\|+\frac{(1-\rho) \alpha_{n}}{1-\gamma_{n}\left(1-s_{n}\right)} \frac{\|f(p)-p\|}{1-\rho} \\
& \leqslant \max \left\{\left\|x_{n}-p\right\|, \frac{\|f(p)-p\|}{1-\rho}\right\} .
\end{aligned}
$$

By induction, we have

$$
\left\|x_{n}-p\right\| \leqslant \max \left\{\left\|x_{1}-p\right\|, \frac{\|f(p)-p\|}{1-\rho}\right\}, \forall n \geqslant 1 .
$$

Hence, $\left\{x_{n}\right\}$ is bounded. Consequently, we deduce immediately that $\left\{f\left(x_{n}\right)\right\}$ and $\left\{T_{t_{n}}\left(s_{n} x_{n}+\left(1-s_{n}\right) x_{n+1}\right)\right\}$ are bounded.

Next, we show that $\lim _{n \rightarrow \infty}\left\|x_{n+1}-x_{n}\right\|=0$. Let $z_{n}=s_{n} x_{n}+\left(1-s_{n}\right) x_{n+1}$ for all $n \geqslant 1$. Then, we have

$$
\begin{aligned}
\left\|z_{n+1}-z_{n}\right\| & =\left\|s_{n+1} x_{n+1}+\left(1-s_{n+1}\right) x_{n+2}-\left(s_{n} x_{n}+\left(1-s_{n}\right) x_{n+1}\right)\right\| \\
& \leqslant\left(1-s_{n+1}\right)\left\|x_{n+2}-x_{n+1}\right\|+s_{n}\left\|x_{n+1}-x_{n}\right\| .
\end{aligned}
$$

Let $y_{n}=\frac{x_{n+1}-\beta_{n} x_{n}}{1-\beta_{n}}$ for all $n \geqslant 1$. Then, we drive that

$$
\begin{aligned}
y_{n+1}-y_{n} & =\frac{x_{n+2}-\beta_{n+1} x_{n+1}}{1-\beta_{n+1}}-\frac{x_{n+1}-\beta_{n} x_{n}}{1-\beta_{n}} \\
& =\frac{\alpha_{n+1} f\left(x_{n+1}\right)+\gamma_{n+1} T_{t_{n+1}} z_{n+1}}{1-\beta_{n+1}}-\frac{\alpha_{n} f\left(x_{n}\right)+\gamma_{n} T_{t_{n}} z_{n}}{1-\beta_{n}} \\
& =\frac{\alpha_{n+1}}{1-\beta_{n+1}} f\left(x_{n+1}\right)-\frac{\alpha_{n}}{1-\beta_{n}} f\left(x_{n}\right)+T_{t_{n+1}} z_{n+1}-T_{t_{n}} z_{n}+\frac{\alpha_{n}}{1-\beta_{n}} T_{t_{n}} z_{n}-\frac{\alpha_{n+1}}{1-\beta_{n+1}} T_{t_{n+1}} z_{n+1} .
\end{aligned}
$$

It follows that

$$
\begin{aligned}
\left\|y_{n+1}-y_{n}\right\| \leqslant & \frac{\alpha_{n+1}}{1-\beta_{n+1}}\left\|f\left(x_{n+1}\right)-T_{t_{n+1}} z_{n+1}\right\|+\frac{\alpha_{n}}{1-\beta_{n}}\left\|T_{t_{n}} z_{n}-f\left(x_{n}\right)\right\|+\left\|T_{t_{n+1}} z_{n+1}-T_{t_{n+1}} z_{n}\right\| \\
& +\left\|T_{t_{n+1}} z_{n}-T_{t_{n}} z_{n}\right\| \\
\leqslant & \frac{\alpha_{n+1}}{1-\beta_{n+1}}\left\|f\left(x_{n+1}\right)-T_{t_{n+1}} z_{n+1}\right\|+\frac{\alpha_{n}}{1-\beta_{n}}\left\|T_{t_{n}} z_{n}-f\left(x_{n}\right)\right\| \\
& +\left(1-s_{n+1}\right)\left\|x_{n+2}-x_{n+1}\right\|+s_{n}\left\|x_{n+1}-x_{n}\right\|+\left\|T_{t_{n+1}} z_{n}-T_{t_{n}} z_{n}\right\| .
\end{aligned}
$$

Now, we estimate $\left\|x_{n+2}-x_{n+1}\right\|$. Observe that

$$
\begin{aligned}
\left\|x_{n+2}-x_{n+1}\right\|= & \left\|\alpha_{n+1} f\left(x_{n+1}\right)+\beta_{n+1} x_{n+1}+\gamma_{n+1} T_{t_{n+1}} z_{n+1}-\left(\alpha_{n} f\left(x_{n}\right)+\beta_{n} x_{n}+\gamma_{n} T_{t_{n}} z_{n}\right)\right\| \\
= & \| \alpha_{n+1}\left(f\left(x_{n+1}\right)-f\left(x_{n}\right)\right)+\left(\alpha_{n+1}-\alpha_{n}\right)\left(f\left(x_{n}\right)-T_{t_{n}} z_{n}\right)+\beta_{n+1}\left(x_{n+1}-x_{n}\right) \\
& +\left(\beta_{n+1}-\beta_{n}\right)\left(x_{n}-T_{t_{n}} z_{n}\right)+\gamma_{n+1}\left(T_{t_{n+1}} z_{n+1}-T_{t_{n}} z_{n}\right) \| \\
\leqslant & \alpha_{n+}\left\|f\left(x_{n+1}\right)-f\left(x_{n}\right)\right\|+\left|\alpha_{n+1}-\alpha_{n}\right|\left\|f\left(x_{n}\right)-T_{t_{n}} z_{n}\right\|+\beta_{n+1}\left\|x_{n+1}-x_{n}\right\| \\
& +\left|\beta_{n+1}-\beta_{n}\right|\left\|x_{n}-T_{t_{n}} z_{n}\right\|+\gamma_{n+1}\left\|T_{t_{n+1}} z_{n+1}-T_{t_{n+1}} z_{n}\right\|+\gamma_{n+1}\left\|T_{t_{n+1}} z_{n}-T_{t_{n}} z_{n}\right\|
\end{aligned}
$$




$$
\begin{aligned}
\leqslant & \left(\alpha_{n+1} \rho+\beta_{n+1}\right)\left\|x_{n+1}-x_{n}\right\|+\left|\alpha_{n+1}-\alpha_{n}\right|\left\|f\left(x_{n}\right)-T_{t_{n}} z_{n}\right\| \\
& +\left|\beta_{n+1}-\beta_{n}\right|\left\|x_{n}-T_{t_{n}} z_{n}\right\| \\
& +\gamma_{n+1}\left(\left(1-s_{n+1}\right)\left\|x_{n+2}-x_{n+1}\right\|+s_{n}\left\|x_{n+1}-x_{n}\right\|\right)+\gamma_{n+1}\left\|T_{t_{n+1}} z_{n}-T_{t_{n}} z_{n}\right\| \\
\leqslant & \left(\alpha_{n+1} \rho+\beta_{n+1}+\gamma_{n+1} s_{n}\right)\left\|x_{n+1}-x_{n}\right\|+\left(\left|\alpha_{n+1}-\alpha_{n}\right|+\left|\beta_{n+1}-\beta_{n}\right|\right) M_{1} \\
& +\gamma_{n+1}\left(1-s_{n+1}\right)\left\|x_{n+2}-x_{n+1}\right\|+\gamma_{n+1}\left\|T_{t_{n+1}} z_{n}-T_{t_{n}} z_{n}\right\|,
\end{aligned}
$$

where $M_{1}=\sup _{n \geqslant 1}\left\{\left\|f\left(x_{n}\right)\right\|+\left\|T_{t_{n}} z_{n}\right\|,\left\|x_{n}\right\|+\left\|T_{t_{n}} z_{n}\right\|\right\}$. It follows that

$$
\begin{aligned}
&\left\|x_{n+2}-x_{n+1}\right\| \\
& \leqslant \frac{\alpha_{n+1} \rho+\beta_{n+1}+\gamma_{n+1} s_{n}}{1-\gamma_{n+1}\left(1-s_{n+1}\right)}\left\|x_{n+1}-x_{n}\right\|+\left(\frac{\left|\alpha_{n+1}-\alpha_{n}\right|}{1-\gamma_{n+1}\left(1-s_{n+1}\right)}+\frac{\left|\beta_{n+1}-\beta_{n}\right|}{1-\gamma_{n+1}\left(1-s_{n+1}\right)}\right) M_{1} \\
&+\frac{\gamma_{n+1}}{1-\gamma_{n+1}\left(1-s_{n+1}\right)}\left\|T_{t_{n+1}} z_{n}-T_{t_{n}} z_{n}\right\| \\
&=\left(1-\frac{(1-\rho) \alpha_{n+1}}{1-\gamma_{n+1}\left(1-s_{n+1}\right)}\right)\left\|x_{n+1}-x_{n}\right\|+\left(\frac{\left|\alpha_{n+1}-\alpha_{n}\right|}{1-\gamma_{n+1}\left(1-s_{n+1}\right)}+\frac{\left|\beta_{n+1}-\beta_{n}\right|}{1-\gamma_{n+1}\left(1-s_{n+1}\right)}\right) M_{1} \\
&+\frac{\gamma_{n+1}}{1-\gamma_{n+1}\left(1-s_{n+1}\right)}\left\|T_{t_{n+1}} z_{n}-T_{t_{n}} z_{n}\right\| .
\end{aligned}
$$

Substituting (3.4) into (3.3), we get that

$$
\begin{aligned}
\left\|y_{n+1}-y_{n}\right\| \leqslant & \frac{\alpha_{n+1}}{1-\beta_{n+1}}\left\|f\left(x_{n+1}\right)-T_{t_{n+1}} z_{n+1}\right\|+\frac{\alpha_{n}}{1-\beta_{n}}\left\|T_{t_{n}} z_{n}-f\left(x_{n}\right)\right\| \\
& +\left(1-s_{n+1}\right)\left\{\left(1-\frac{(1-\rho) \alpha_{n+1}}{1-\gamma_{n+1}\left(1-s_{n+1}\right)}\right)\left\|x_{n+1}-x_{n}\right\|\right. \\
& +\left(\frac{\left|\alpha_{n+1}-\alpha_{n}\right|}{1-\gamma_{n+1}\left(1-s_{n+1}\right)}+\frac{\left|\beta_{n+1}-\beta_{n}\right|}{1-\gamma_{n+1}\left(1-s_{n+1}\right)}\right) M_{1} \\
& \left.+\frac{\gamma_{n+1}}{1-\gamma_{n+1}\left(1-s_{n+1}\right)}\left\|T_{t_{n+1}} z_{n}-T_{t_{n}} z_{n}\right\|\right\} \\
& +s_{n}\left\|x_{n+1}-x_{n}\right\|+\left\|T_{t_{n+1}} z_{n}-T_{t_{n}} z_{n}\right\| \\
\leqslant & \left(1-\frac{(1-\rho) \alpha_{n+1}\left(1-s_{n+1}\right)}{1-\gamma_{n+1}\left(1-s_{n+1}\right)}\right)\left\|x_{n+1}-x_{n}\right\|+\frac{\alpha_{n+1}}{1-\beta_{n+1}}\left\|f\left(x_{n+1}\right)-T_{t_{n+1}} z_{n+1}\right\| \\
& +\frac{\alpha_{n}}{1-\beta_{n}}\left\|T_{t_{n}} z_{n}-f\left(x_{n}\right)\right\|+\left(\frac{\left|\alpha_{n+1}-\alpha_{n}\right|}{1-\gamma_{n+1}\left(1-s_{n+1}\right)}+\frac{\left|\beta_{n+1}-\beta_{n}\right|}{1-\gamma_{n+1}\left(1-s_{n+1}\right)}\right) M_{1} \\
& +\frac{1}{1-\gamma_{n+1}\left(1-s_{n+1}\right)}\left\|T_{t_{n+1}} z_{n}-T_{t_{n}} z_{n}\right\| .
\end{aligned}
$$

Since $t_{n+1}=h+t_{n}$ for all $h \geqslant 0$, we have

$$
\lim _{n \rightarrow \infty}\left\|T_{t_{n+1}} z_{n}-T_{t_{n}} z_{n}\right\|=\lim _{n \rightarrow \infty}\left\|T_{h} T_{t_{n}} z_{n}-T_{t_{n}} z_{n}\right\| \leqslant \lim _{n \rightarrow \infty} \sup _{x \in\left\{z_{n}\right\}}\left\|T_{h} T_{t_{n}} x-T_{t_{n}} x\right\|=0 .
$$

Then from (3.5), we have

$$
\limsup _{n \rightarrow \infty}\left(\left\|y_{n+1}-y_{n}\right\|-\left\|x_{n+1}-x_{n}\right\|\right) \leqslant 0
$$

By Lemma 2.12, we have

$$
\lim _{n \rightarrow \infty}\left\|y_{n}-x_{n}\right\|=0
$$

Consequently, we have

$$
\lim _{n \rightarrow \infty}\left\|x_{n+1}-x_{n}\right\|=\lim _{n \rightarrow \infty}\left(1-\beta_{n}\right)\left\|y_{n}-x_{n}\right\|=0
$$


and

$$
\lim _{n \rightarrow \infty}\left\|z_{n}-x_{n}\right\|=\lim _{n \rightarrow \infty}\left(1-s_{n}\right)\left\|x_{n+1}-x_{n}\right\|=0 .
$$

Next, we show that $\lim _{n \rightarrow \infty}\left\|x_{n}-T_{h} x_{n}\right\|=0$ for all $h \geqslant 0$. Since

$$
\begin{aligned}
\left\|x_{n+1}-T_{t_{n}} x_{n}\right\| & \leqslant \alpha_{n}\left\|f\left(x_{n}\right)-T_{t_{n}} z_{n}\right\|+\beta_{n}\left\|x_{n}-T_{t_{n}} z_{n}\right\|+\gamma_{n}\left\|T_{t_{n}} z_{n}-T_{t_{n}} x_{n}\right\| \\
& \leqslant \alpha_{n}\left\|f\left(x_{n}\right)-T_{t_{n}} x_{n}\right\|+\beta_{n}\left\|x_{n}-x_{n+1}\right\|+\beta_{n}\left\|x_{n+1}-T_{t_{n}} x_{n}\right\|+\gamma_{n}\left\|z_{n}-x_{n}\right\|,
\end{aligned}
$$

and hence

$$
\left\|x_{n+1}-T_{t_{n}} x_{n}\right\| \leqslant \frac{\alpha_{n}}{1-\beta_{n}}\left\|f\left(x_{n}\right)-T_{t_{n}} x_{n}\right\|+\frac{\beta_{n}}{1-\beta_{n}}\left\|x_{n+1}-x_{n}\right\|+\frac{\gamma_{n}}{1-\beta_{n}}\left\|z_{n}-x_{n}\right\| \rightarrow 0 \text { as } n \rightarrow \infty .
$$

Consequently,

$$
\begin{aligned}
\left\|z_{n}-T_{t_{n}} z_{n}\right\| & \leqslant\left\|z_{n}-x_{n+1}\right\|+\left\|x_{n+1}-T_{t_{n}} z_{n}\right\| \\
& \leqslant s_{n}\left\|x_{n}-x_{n+1}\right\|+\left\|x_{n+1}-T_{t_{n}} x_{n}\right\|+\left\|T_{t_{n}} x_{n}-T_{t_{n}} z_{n}\right\| \\
& \leqslant s_{n}\left\|x_{n}-x_{n+1}\right\|+\left\|x_{n+1}-T_{t_{n}} x_{n}\right\|+\left\|x_{n}-z_{n}\right\| \rightarrow 0 \text { as } n \rightarrow \infty .
\end{aligned}
$$

Then, for all $h \geqslant 0$, we obtain that

$$
\begin{aligned}
\left\|z_{n}-T_{h} z_{n}\right\| & \leqslant\left\|z_{n}-T_{t_{n}} z_{n}\right\|+\left\|T_{t_{n}} z_{n}-T_{h} T_{t_{n}} z_{n}\right\|+\left\|T_{h} T_{t_{n}} z_{n}-T_{h} z_{n}\right\| \\
& \leqslant 2\left\|z_{n}-T_{t_{n}} z_{n}\right\|+\sup _{x \in\left\{z_{n}\right\}}\left\|T_{t_{n}} x-T_{h} T_{t_{n}} x\right\| \rightarrow 0 \text { as } n \rightarrow \infty .
\end{aligned}
$$

From (3.6), we also have

$$
\lim _{n \rightarrow \infty}\left\|x_{n}-T_{h} x_{n}\right\|=0, \quad \forall h \geqslant 0 .
$$

Let $u_{m}=\alpha_{m} f\left(u_{m}\right)+\left(1-\alpha_{m}\right) T_{t_{m}} u_{m}$, where $\left\{\alpha_{m}\right\}$ and $\left\{t_{m}\right\}$ satisfy the condition of Theorem 2.11. From these, we know that $\left\{u_{m}\right\}$ converges strongly to $p$, where $p \in F(\mathcal{S})$ is a unique solution of (3.2). Since

$$
\begin{aligned}
\left\|u_{m}-x_{n}\right\| \varphi\left(\left\|u_{m}-x_{n}\right\|\right)= & \alpha_{n}\left\langle f\left(u_{m}\right)-x_{n}, j_{\varphi}\left(u_{m}-x_{n}\right)\right\rangle+\left(1-\alpha_{m}\right)\left\langle T_{t_{m}} u_{m}-x_{n}, j_{\varphi}\left(u_{m}-x_{n}\right)\right\rangle \\
= & \alpha_{m}\left\langle f\left(u_{m}\right)-f(p)-u_{m}+p, j_{\varphi}\left(u_{m}-x_{n}\right)\right\rangle+\alpha_{m}\left\langle f(p)-p, j_{\varphi}\left(u_{m}-x_{n}\right)\right\rangle \\
& +\alpha_{m}\left\langle u_{m}-x_{n}, j_{\varphi}\left(u_{m}-x_{n}\right)\right\rangle+\left(1-\alpha_{m}\right)\left\langle T_{t_{m}} u_{m}-T_{t_{m}} x_{n}, j_{\varphi}\left(u_{m}-x_{n}\right)\right\rangle \\
& +\left(1-\alpha_{m}\right)\left\langle T_{t_{m}} x_{n}-x_{n}, j_{\varphi}\left(u_{m}-x_{n}\right)\right\rangle \\
\leqslant & \left\|u_{m}-x_{n}\right\| \varphi\left(\left\|u_{m}-x_{n}\right\|\right)+\left\|T_{t_{m}} x_{n}-x_{n}\right\| \varphi\left(\left\|u_{m}-x_{n}\right\|\right) \\
& +\alpha_{m}(1+\rho) \varphi\left(\left\|u_{m}-x_{n}\right\|\right)\left\|u_{m}-p\right\|+\alpha_{m}\left\langle f(p)-p, j_{\varphi}\left(u_{m}-x_{n}\right)\right\rangle,
\end{aligned}
$$

which implies that

$$
\left\langle f(p)-p, j_{\varphi}\left(x_{n}-u_{m}\right)\right\rangle \leqslant\left(\frac{\left\|T_{t_{m}} x_{n}-x_{n}\right\|}{\alpha_{m}}+(1+\rho)\left\|u_{m}-p\right\|\right) M_{2}
$$

where $M_{2}=\sup _{n \geqslant 1}\left\{\varphi\left(\left\|u_{m}-x_{n}\right\|\right)\right\}$. Now, taking the upper limit as $n \rightarrow \infty$ and as $m \rightarrow \infty$, respectively in (3.7), we obtain

$$
\limsup _{m \rightarrow \infty} \limsup _{n \rightarrow \infty}\left\langle f(p)-p, j_{\varphi}\left(x_{n}-u_{m}\right)\right\rangle \leqslant 0 .
$$

Since $j_{\varphi}$ is norm-weak* uniformly continuous on bounded sets, as $m \rightarrow \infty$, then

$$
\left\langle f(p)-p, j_{\varphi}\left(x_{n}-u_{m}\right)\right\rangle \rightarrow\left\langle f(p)-p, j_{\varphi}\left(x_{n}-p\right)\right\rangle .
$$


Hence, for each $\epsilon>0$, there exists $N \geqslant 1$ such that if $m>N$, for all $n \geqslant 1$ we have

$$
\left\langle f(p)-p, j_{\varphi}\left(x_{n}-p\right)\right\rangle<\left\langle f(p)-p, j_{\varphi}\left(x_{n}-u_{m}\right)\right\rangle+\epsilon .
$$

Thus taking upper limit as $n \rightarrow \infty$ and as $m \rightarrow \infty$ in both sides of (3.9), we get from (3.8) that

$$
\limsup _{n \rightarrow \infty}\left\langle f(p)-p, j_{\varphi}\left(x_{n}-p\right)\right\rangle \leqslant \epsilon
$$

Since $\epsilon>0$ is arbitrary, then we obtain

$$
\limsup _{n \rightarrow \infty}\left\langle f(p)-p, j_{\varphi}\left(x_{n}-p\right)\right\rangle \leqslant 0
$$

Finally, we show that $x_{n}$ converges strongly to $p$. We have from Lemma 2.4 that

$$
\begin{aligned}
\Phi\left(\left\|x_{n+1}-p\right\|\right)= & \Phi\left(\| \alpha_{n}\left(f\left(x_{n}\right)-p\right)+\beta_{n}\left(x_{n}-p\right)+\gamma_{n}\left(T_{t_{n}}\left(s_{n} x_{n}+\left(1-s_{n}\right) x_{n+1}\right)-p \|\right)\right) \\
\leqslant & \Phi\left(\left\|\alpha_{n}\left(f\left(x_{n}\right)-f(p)\right)+\beta_{n}\left(x_{n}-p\right)+\gamma_{n}\left(T_{t_{n}}\left(s_{n} x_{n}+\left(1-s_{n}\right) x_{n+1}\right)-p\right)\right\|\right) \\
& +\alpha_{n}\left\langle f(p)-p, j_{\varphi}\left(x_{n+1}-p\right)\right\rangle \\
\leqslant & \alpha_{n} \Phi\left(\left\|f\left(x_{n}\right)-f(p)\right\|\right)+\beta_{n} \Phi\left(\left\|x_{n}-p\right\|\right)+\gamma_{n} \Phi\left(\left\|T_{t_{n}}\left(s_{n} x_{n}+\left(1-s_{n}\right) x_{n+1}\right)-p\right\|\right) \\
& +\alpha_{n}\left\langle f(p)-p, j_{\varphi}\left(x_{n+1}-p\right)\right\rangle \\
\leqslant & \alpha_{n} \rho \Phi\left(\left\|x_{n}-p\right\|\right)+\beta_{n} \Phi\left(\left\|x_{n}-p\right\|\right)+\gamma_{n} \Phi\left(\left\|s_{n}\left(x_{n}-p\right)+\left(1-s_{n}\right)\left(x_{n+1}-p\right)\right\|\right) \\
& +\alpha_{n}\left\langle f(p)-p, j_{\varphi}\left(x_{n+1}-p\right)\right\rangle \\
\leqslant & \alpha_{n} \rho \Phi\left(\left\|x_{n}-p\right\|\right)+\beta_{n} \Phi\left(\left\|x_{n}-p\right\|\right)+\gamma_{n}\left(s_{n} \Phi\left(\left\|x_{n}-p\right\|\right)+\left(1-s_{n}\right) \Phi\left(\left\|x_{n+1}-p\right\|\right)\right) \\
& +\alpha_{n}\left\langle f(p)-p, j_{\varphi}\left(x_{n+1}-p\right)\right\rangle \\
= & \left(\alpha_{n} \rho+\beta_{n}+\gamma_{n} s_{n}\right) \Phi\left(\left\|x_{n}-p\right\|\right)+\gamma_{n}\left(1-s_{n}\right) \Phi\left(\left\|x_{n+1}-p\right\|\right) \\
& +\alpha_{n}\left\langle f(p)-p, j_{\varphi}\left(x_{n+1}-p\right)\right\rangle,
\end{aligned}
$$

which implies that

$$
\begin{aligned}
\Phi\left(\left\|x_{n+1}-p\right\|\right) & \leqslant \frac{\alpha_{n} \rho+\beta_{n}+\gamma_{n} s_{n}}{1-\gamma_{n}\left(1-s_{n}\right)} \Phi\left(\left\|x_{n}-p\right\|\right)+\frac{\alpha_{n}}{\gamma_{n}\left(1-s_{n}\right)}\left\langle f(p)-p, j_{\varphi}\left(x_{n+1}-p\right)\right\rangle \\
& =\left(1-\frac{(1-\rho) \alpha_{n}}{1-\gamma_{n}\left(1-s_{n}\right)}\right) \Phi\left(\left\|x_{n}-p\right\|\right)+\frac{\alpha_{n}}{\gamma_{n}\left(1-s_{n}\right)}\left\langle f(p)-p, j_{\varphi}\left(x_{n+1}-p\right)\right\rangle \\
& =\left(1-\theta_{n}\right) \Phi\left(\left\|x_{n}-p\right\|\right)+\theta_{n} \sigma_{n},
\end{aligned}
$$

where $\theta_{n}=\frac{(1-\rho) \alpha_{n}}{1-\gamma_{n}\left(1-s_{n}\right)}$ and $\sigma_{n}=\frac{1}{1-\rho}\left\langle f(p)-p, j_{\varphi}\left(x_{n+1}-p\right)\right\rangle$. From (C1) and (3.10), we see that $\sum_{n=1}^{\infty} \theta_{n}=\infty$ and $\limsup _{n \rightarrow \infty} \sigma_{n} \leqslant 0$. We conclude by Lemma 2.13 that $\Phi\left(\left\|x_{n}-p\right\|\right) \rightarrow 0$ as $n \rightarrow \infty$. By the property of $\Phi$, we obtain that $\left\{x_{n}\right\}$ converges strongly to $p$ as $n \rightarrow \infty$. This completes the proof.

Corollary 3.2. Let $\mathrm{C}$ be a nonempty, closed, and convex subset of a real Hilbert space $\mathrm{H}$. Let $\mathcal{S}=\left\{\mathrm{T}_{\mathrm{t}}\right\}_{\mathrm{t} \geqslant 0}: \mathrm{C} \rightarrow \mathrm{C}$ be a u.a.r. nonexpansive semigroup such that $\mathrm{F}(\mathcal{S}):=\bigcap_{\mathrm{t} \geqslant 0} \mathrm{~F}\left(\mathrm{~T}_{\mathrm{t}}\right) \neq \emptyset$ and $\mathrm{f}$ be a contraction on $\mathrm{C}$ with coefficient $\rho \in(0,1)$. For given $x_{1} \in C$, let $\left\{x_{n}\right\}$ be a sequence generated by

$$
x_{n+1}=\alpha_{n} f\left(x_{n}\right)+\beta_{n} x_{n}+\gamma_{n} T_{t_{n}}\left(s_{n} x_{n}+\left(1-s_{n}\right) x_{n+1}\right), \quad \forall n \geqslant 1 .
$$

Suppose that $\left\{\alpha_{n}\right\},\left\{\beta_{n}\right\},\left\{\gamma_{n}\right\},\left\{s_{n}\right\}$, and $\left\{t_{n}\right\}$ be the same as in Theorem 3.1. Then, $\left\{x_{n}\right\}$ defined by (3.11) converges strongly to a point $\mathrm{p} \in \mathrm{F}(\mathcal{S})$, which also solves the variational inequality

$$
\left\langle f(p)-x^{*}, z-p\right\rangle \leqslant 0, \forall z \in F(\mathcal{S}) .
$$

\section{Some applications}

4.1. Convergence theorem for a family of mappings

Definition 4.1. Let $C$ be a subset of a Banach space $E$. Let $\left\{T_{n}\right\}_{n=1}^{\infty}: C \rightarrow C$ be a family of mappings such 
that $\bigcap_{n=1}^{\infty} F\left(T_{n}\right) \neq \emptyset$. We say that $\left\{T_{\mathfrak{n}}\right\}_{\mathfrak{n}=1}^{\infty}$ satisfies the AKTT-condition ([3]) if

$$
\sum_{n=1}^{\infty} \sup _{x \in C}\left\|T_{n+1} x-T_{n} x\right\|<\infty .
$$

Lemma 4.2 ([3]). Suppose that $\left\{T_{n}\right\}_{n=1}^{\infty}$ satisfy the AKTT-condition. Then, for any $x \in C,\left\{T_{n} x\right\}_{n=1}^{\infty}$ converges strongly to some point of $\mathrm{C}$. Further, let $\mathrm{T}: \mathrm{C} \rightarrow \mathrm{C}$ defined by $\mathrm{Tx}=\lim _{\mathrm{n} \rightarrow \infty} \mathrm{T}_{\mathrm{n}} \mathrm{x}$ for all $\mathrm{x} \in \mathrm{C}$. Then, $\lim _{n \rightarrow \infty} \sup _{x \in C}\left\|T x-T_{n} x\right\|=0$.

In the sequel, we say that $\left(\left\{T_{n}\right\}_{\mathfrak{n}=1}^{\infty}, T\right)$ satisfies the AKTT-condition if $\left\{T_{n}\right\}_{n=1}^{\infty}$ satisfies the AKTTcondition and $T$ is defined by (4.1) with $\bigcap_{n=1}^{\infty} F\left(T_{n}\right)=F(T)$.

Theorem 4.3. Let $\mathrm{C}$ be a nonempty, closed, and convex subset of a real reflexive strictly convex Banach space $\mathrm{E}$, which has a uniformly Gâteaux differentiable norm and admits the duality mapping $j_{\varphi}$. Let $\left\{\mathrm{T}_{\mathrm{n}}\right\}_{\mathfrak{n}=1}^{\infty}: \mathrm{C} \rightarrow \mathrm{C}$ be a sequence of nonexpansive mappings such that $\bigcap_{n=1}^{\infty} \mathrm{F}\left(\mathrm{T}_{n}\right) \neq \emptyset$ and $\mathrm{f}$ be a contraction on $\mathrm{C}$ with coefficient $\rho \in(0,1)$. For given $x_{1} \in C$, let $\left\{x_{n}\right\}$ be a sequence generated by

$$
x_{n+1}=\alpha_{n} f\left(x_{n}\right)+\beta_{n} x_{n}+\gamma_{n} T_{n}\left(s_{n} x_{n}+\left(1-s_{n}\right) x_{n+1}\right), \quad \forall n \geqslant 1 .
$$

Suppose that $\left\{\alpha_{n}\right\},\left\{\beta_{n}\right\},\left\{\gamma_{n}\right\}$, and $\left\{s_{n}\right\}$ be the same as in Theorem 3.1. Suppose in addition, $\left(\left\{T_{n}\right\}_{n=1}^{\infty}, T\right)$ satisfies the AKTT-condition. Then, $\left\{x_{n}\right\}$ defined by (4.2) converges strongly to a point $p \in \bigcap_{n=1}^{\infty} F\left(T_{n}\right)$, which also solves the variational inequality

$$
\left\langle f(p)-p, j_{\varphi}(z-p)\right\rangle \leqslant 0, \quad \forall z \in \bigcap_{n=1}^{\infty} F\left(T_{n}\right) .
$$

Proof. Following the proof line as in Theorem 3.1, we can show that $\left\{x_{n}\right\}$ is bounded and $\lim _{n \rightarrow \infty} \| x_{n}-$ $\mathrm{T}_{n} x_{n} \|=0$. Since $\left(\left\{\mathrm{T}_{n}\right\}_{n=1}^{\infty}, T\right)$ satisfies the AKTT-condition, we obtain from Lemma 4.2 that

$$
\begin{aligned}
\left\|\mathrm{T}_{\mathrm{n}+1} z_{\mathrm{n}}-\mathrm{T}_{\mathrm{n}} z_{\mathrm{n}}\right\| & =\left\|\mathrm{T}_{\mathrm{n}+1} z_{\mathrm{n}}-\mathrm{T} z_{\mathrm{n}}\right\|+\left\|\mathrm{T} z_{\mathrm{n}}-\mathrm{T}_{\mathrm{n}} z_{\mathrm{n}}\right\| \\
& \leqslant \sup _{x \in\left\{z_{n}\right\}}\left\|\mathrm{T}_{\mathrm{n}+1} x-\mathrm{T} x\right\|+\sup _{x \in\left\{z_{\mathrm{n}}\right\}}\left\|\mathrm{T} x-\mathrm{T}_{\mathrm{n}} x\right\| \rightarrow 0 \text { as } \mathrm{n} \rightarrow \infty .
\end{aligned}
$$

On the other hand, we need to show that $\lim _{n \rightarrow \infty}\left\|x_{n}-T x_{n}\right\|=0$. Again, since $\left(\left\{T_{n}\right\}_{n=1}^{\infty}, T\right)$ satisfies the AKTT-condition, then we obtain that

$$
\left\|x_{n}-T x_{n}\right\| \leqslant\left\|x_{n}-T_{n} x_{n}\right\|+\left\|T_{n} x_{n}-T x_{n}\right\| \leqslant\left\|x_{n}-T_{n} x_{n}\right\|+\sup _{x \in\left\{x_{n}\right\}}\left\|T_{n} x-T x\right\| \rightarrow 0 \text { as } n \rightarrow \infty .
$$

Some parts of the proof are also the same as the Theorem 3.1. Then, we can obtain the desired conclusion easily. This completes the proof.

Example 4.4. Let $C=E=\mathbb{R}$ with the usual norm. For each $n \geqslant 1$, define $T_{n}$ by

$$
\mathrm{T}_{\mathrm{n}} x= \begin{cases}0, & x=0, \\ \sin x+\frac{1}{n^{2},} & x \neq 0\end{cases}
$$

for all $x \in C$. It is not hard to show that $\left\{T_{n}\right\}_{n=1}^{\infty}$ is nonexpansive and satisfies the AKTT-condition with $\bigcap_{n=1}^{\infty} F\left(T_{n}\right)=F(T)=\{0\}$, where $T x=\lim _{n \rightarrow \infty} T_{n} x$ for all $x \in C$.

\subsection{The problem of finding zeros of accretive operators}

Let $A \subset E \times E$ be an operator. We denote by $D(A)$ and $\overline{D(A)}$ the domain of $A$ and closure of $D(A)$, respectively. We say that $A$ is said to be accretive if there exists $j_{\varphi}\left(x_{1}-x_{2}\right) \in J_{\varphi}\left(x_{1}-x_{2}\right)$ such that $\left\langle y_{1}-y_{2}, j_{\varphi}\right\rangle \geqslant 0$, where $\left(x_{i}, y_{i}\right) \in A$ for $i=1,2$. We say that $A$ is said to satisfy the range condition 
if $\overline{D(A)}=R(I+\lambda A)$ for all $\lambda>0$, where $R(I+\lambda A)$ is the range of $I+\lambda A$. It is well known that if $A$ is an accretive operator which satisfies the range condition, then we can defined a single-valued mapping $\mathrm{J}_{\lambda}^{A}: R(I+\lambda A) \rightarrow D(A)$ by $\mathrm{J}_{\lambda}=(\mathrm{I}+\lambda A)^{-1}$, which is called the resolvent of $A$. We denote by $A^{-1} 0$ the set of zeros of $A$, i.e., $A^{-1} 0=\{x \in D(A): 0 \in A x\}$. It is well known that $J_{\lambda}$ is nonexpansive and $F\left(J_{\lambda}\right)=A^{-1} 0$ (see [27]). We also know the following [16]: For each $\lambda, \mu>0$ and $x \in R(I+\lambda A) \cap R(I+\mu A)$, it holds that

$$
\left\|\mathrm{J}_{\lambda} x-\mathrm{J}_{\mu} \mathrm{x}\right\| \leqslant \frac{|\lambda-\mu|}{\lambda}\left\|x-\mathrm{J}_{\lambda} \mathrm{x}\right\|
$$

Lemma 4.5 ([3]). Let $\mathrm{C}$ be a nonempty, closed, and convex subset of a Banach space $\mathrm{E}$. Let $\mathrm{A} \subset \mathrm{E} \times \mathrm{E}$ be an accretive operator such that $A^{-1} 0 \neq \emptyset$ which satisfies the condition $\overline{\mathrm{D}(A)} \subset \mathrm{C} \subset \bigcap_{\lambda>0} \mathrm{R}(\mathrm{I}+\lambda \mathrm{A})$. Suppose that $\left\{\lambda_{n}\right\} \subset(0, \infty)$ such that $\inf \left\{\lambda_{n}: n \in \mathbb{N}\right\}>0$ and $\lim _{n \rightarrow \infty}\left|\lambda_{n+1}-\lambda_{n}\right|=0$. Then $\left\{J_{\lambda_{n}}\right\}$ satisfies the AKTTcondition. Consequently, for each $x \in \mathrm{C},\left\{\mathrm{J}_{\lambda_{n}} x\right\}$ converges strongly to some point of $\mathrm{C}$. Moreover, let $\mathrm{J}_{\lambda}: \mathrm{C} \rightarrow \mathrm{C}$ defined by $\mathrm{J}_{\lambda} x=\lim _{n \rightarrow \infty} \mathrm{J}_{\lambda_{n}} x$ for all $\mathrm{x} \in \mathrm{C}$ and $\mathrm{F}\left(\mathrm{J}_{\lambda}\right)=\bigcap_{\mathrm{n}=1}^{\infty} \mathrm{F}\left(\mathrm{J}_{\lambda_{\mathrm{n}}}\right)$, where $\lambda_{\mathrm{n}} \rightarrow \lambda$ as $\mathrm{n} \rightarrow \infty$. Then, $\lim _{n \rightarrow \infty} \sup _{x \in C}\left\|J_{\lambda} x-J_{\lambda_{n}} x\right\|=0$.

Theorem 4.6. Let $\mathrm{C}$ be a nonempty, closed, and convex subset of a real reflexive strictly convex Banach space $\mathrm{E}$, which has a uniformly Gâteaux differentiable norm and admits the duality mapping $j_{\varphi}$. Let $\mathrm{A} \subset \mathrm{E} \times \mathrm{E}$ be an accretive operator such that $A^{-1} 0 \neq \emptyset$ which satisfies the condition $\overline{D(A)} \subset \mathrm{C} \subset \bigcap_{\lambda>0} \mathrm{R}(\mathrm{I}+\lambda \mathrm{A})$ and $\mathrm{f}$ be a contraction on $C$ with coefficient $\rho \in(0,1)$. For given $x_{1} \in C$, let $\left\{x_{n}\right\}$ be a sequence generated by

$$
x_{n+1}=\alpha_{n} f\left(x_{n}\right)+\beta_{n} x_{n}+\gamma_{n} J_{\lambda_{n}}\left(s_{n} x_{n}+\left(1-s_{n}\right) x_{n+1}\right), \quad \forall n \geqslant 1,
$$

where $\left\{\lambda_{n}\right\}$ is a real sequence in $(0, \infty)$ with $\inf \left\{\lambda_{n}: n \in \mathbb{N}\right\}>0$ and $\lim _{n \rightarrow \infty}\left|\lambda_{n+1}-\lambda_{n}\right|=0$. Suppose that $\left\{\alpha_{n}\right\},\left\{\beta_{n}\right\},\left\{\gamma_{n}\right\},\left\{s_{n}\right\}$ be the same as in Theorem 3.1. Then $\left\{x_{n}\right\}$ defined by (4.3) converges strongly to a point $p \in A^{-1} 0$, which also solves the variational inequality

$$
\left\langle\mathrm{f}(\mathrm{p})-\mathrm{p}, \dot{j}_{\varphi}(z-\mathrm{p})\right\rangle \leqslant 0, \quad \forall z \in \mathrm{A}^{-1}(0) .
$$

Proof. Since $\left(\left\{\mathrm{J}_{\lambda_{n}}\right\}, \mathrm{J}_{\lambda}\right)$ satisfies the AKTT-condition, by following the proof line in Theorem 4.3, we can conclude the desired conclusion immediately.

\section{Numerical examples}

In this section, we present two numerical experiments to support the main result.

Example 5.1. Let $E=C=\mathbb{R}^{2}, \mathbf{x}=\left(\begin{array}{l}x_{1} \\ x_{2}\end{array}\right) \in \mathbb{R}^{2}$, and $\mathbf{y}=\left(\begin{array}{l}y_{1} \\ y_{2}\end{array}\right) \in \mathbb{R}^{2}$, where $x_{i}, y_{i} \in \mathbb{R}$ for $i=1$, 2. Let $\langle\cdot, \cdot\rangle: \mathbb{R}^{2} \times \mathbb{R}^{2} \rightarrow \mathbb{R}$ be the inner product defined by $\mathbf{x} \cdot \mathbf{y}=x_{1} \mathrm{y}_{1}+x_{2} y_{2}$ and let $\|\cdot\|: \mathbb{R}^{2} \rightarrow \mathbb{R}$ be the usual norm defined by $\|\mathbf{x}\|=\sqrt{x_{1}^{2}+x_{2}^{2}}$. Let $f: \mathbb{R}^{2} \rightarrow \mathbb{R}^{2}$ defined by $f(\mathbf{x})=\frac{1}{4} \mathbf{x}$. For each $t \geqslant 0$, let $T_{t}: \mathbb{R}^{2} \rightarrow \mathbb{R}^{2}$ be a u.a.r. nonexpansive semigroup defined by

$$
\mathrm{T}_{\mathrm{t}} \mathbf{x}=\left(\begin{array}{cc}
\mathrm{e}^{-2 \mathrm{t}} & 0 \\
0 & 1
\end{array}\right) \mathbf{x}
$$

It is not hard to see that $\bigcap_{t \geqslant 0} F\left(T_{t}\right)=p=\left(\begin{array}{c}0 \\ x_{2}\end{array}\right)$. Let $t_{n}=\frac{n}{2}, s_{n}=\frac{n}{n+1}, \alpha_{n}=\frac{1}{50 n+1}, \beta_{n}=\frac{n}{50 n+1}$, we have $\gamma_{n}=\frac{49 n}{50 n+1}$. So our algorithm (3.1) has the following form:

$$
\left(\begin{array}{l}
x_{1}^{n+1} \\
x_{2}^{\mathfrak{n}+1}
\end{array}\right)=\frac{5 n+1}{200 n+4}\left(\begin{array}{l}
x_{1}^{n} \\
x_{2}^{n}
\end{array}\right)+\frac{49 n}{50 n+1}\left(\begin{array}{cc}
e^{-n} & 0 \\
0 & 1
\end{array}\right)\left[\frac{n}{n+1}\left(\begin{array}{l}
x_{1}^{n} \\
x_{2}^{n}
\end{array}\right)+\frac{1}{n+1}\left(\begin{array}{l}
x_{1}^{n+1} \\
x_{2}^{n+1}
\end{array}\right)\right], \forall n \geqslant 1 .
$$

Figure 1.

Choose $\mathbf{x}_{\mathbf{1}}=\left(\begin{array}{l}2 \\ 3\end{array}\right)$ be the initial point. Then, we obtain the numerical results shown in Table 1 and 
Table 1: Numerical results of Example 5.1.

\begin{tabular}{|c|c|c|}
\hline $\mathrm{n}$ & $\mathbf{x}^{\mathrm{n}}=\left(\mathrm{x}_{1}^{\mathfrak{n}}, x_{2}^{\mathfrak{n}}\right)^{\top}$ & $\left\|\mathbf{x}_{\mathrm{n}}-\mathrm{p}\right\|$ \\
\hline 1 & $(2.00000000,3.00000000)^{\top}$ & 2.00000000 \\
2 & $(0.49707287,3.00000000)^{\mathrm{T}}$ & 0.49707287 \\
3 & $(0.05745620,3.00000000)^{\mathrm{T}}$ & 0.05745620 \\
4 & $(0.00337702,3.00000000)^{\top}$ & 0.00337702 \\
5 & $(0.00012027,3.00000000)^{\mathrm{T}}$ & 0.00012027 \\
6 & $(3.18033 \mathrm{e}-06,3.00000000)^{\top}$ & $3.18033 \mathrm{e}-06$ \\
7 & $(7.26788 \mathrm{e}-08,3.00000000)^{\top}$ & $7.26788 \mathrm{e}-08$ \\
8 & $(1.55817 \mathrm{e}-09,3.00000000)^{\top}$ & $1.55817 \mathrm{e}-09$ \\
9 & $(3.25133 \mathrm{e}-11,3.00000000)^{\top}$ & $3.25133 \mathrm{e}-11$ \\
10 & $(6.70392 \mathrm{e}-13,3.00000000)^{\top}$ & $6.70392 \mathrm{e}-13$ \\
\hline
\end{tabular}

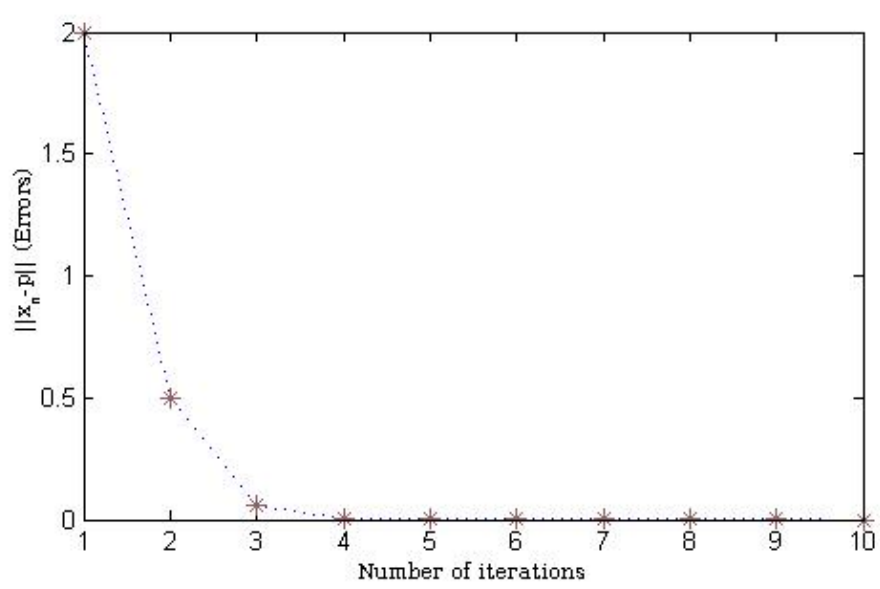

Figure 1: Behavior of convergence error values.

Example 5.2. Let $E=C=\mathbb{R}^{3}, \mathbf{x}=\left(\begin{array}{l}x_{1} \\ x_{2} \\ x_{3}\end{array}\right) \in \mathbb{R}^{3}$, and $\mathbf{y}=\left(\begin{array}{l}y_{1} \\ y_{2} \\ y_{3}\end{array}\right) \in \mathbb{R}^{3}$, where $x_{i}, y_{i} \in \mathbb{R}$ for $i=1,2,3$. Let $\langle\cdot, \cdot\rangle: \mathbb{R}^{3} \times \mathbb{R}^{3} \rightarrow \mathbb{R}$ be the inner product defined by $\mathbf{x} \cdot \mathbf{y}=x_{1} y_{1}+x_{2} y_{2}+x_{3} y_{3}$ and let $\|\cdot\|: \mathbb{R}^{3} \rightarrow \mathbb{R}$ be the usual norm defined by $\|\mathbf{x}\|=\sqrt{x_{1}^{2}+x_{2}^{2}+x_{3}^{2}}$. Let $f: \mathbb{R}^{3} \rightarrow \mathbb{R}^{3}$ defined by $f(\mathbf{x})=\frac{1}{2} \mathbf{x}$. For each $t \geqslant 0$, let $T_{t}: \mathbb{R}^{3} \rightarrow \mathbb{R}^{3}$ be a u.a.r. nonexpansive semigroup defined by

$$
T_{t} x=e^{-t}\left(\begin{array}{ccc}
\cos \sqrt{2} t & \sin \sqrt{2} t & 0 \\
-\sin \sqrt{2} t & \cos \sqrt{2} t & 0 \\
0 & 0 & 1
\end{array}\right) \mathbf{x}
$$

It is not hard to see that $\bigcap_{t \geqslant 0} F\left(T_{t}\right)=p=\left(\begin{array}{l}0 \\ 0 \\ 0\end{array}\right)$. Let $t_{n}=2 n, s_{n}=\frac{1}{2}, \alpha_{n}=\frac{1}{2 n+1}, \beta_{n}=\frac{n}{2 n+1}$, we have $\gamma_{\mathrm{n}}=\frac{\mathrm{n}}{2 \mathrm{n}+1}$. So our algorithm (3.1) has the following form:

$$
\left(\begin{array}{l}
x_{1}^{n+1} \\
x_{2}^{n+1} \\
x_{3}^{n+1}
\end{array}\right)=\left(\begin{array}{l}
0.5 x_{1}^{n} \\
0.5 x_{2}^{n} \\
0.5 x_{3}^{n}
\end{array}\right)+\frac{n e^{-2 n}}{2 n+1}\left(\begin{array}{ccc}
\cos 2 \sqrt{2} n & \sin 2 \sqrt{2} n & 0 \\
-\sin 2 \sqrt{2} n & \cos 2 \sqrt{2} n & 0 \\
0 & 0 & 1
\end{array}\right)\left[\left(\begin{array}{l}
0.5 x_{1}^{n} \\
0.5 x_{2}^{n} \\
0.5 x_{3}^{n}
\end{array}\right)+\left(\begin{array}{l}
0.5 x_{1}^{n+1} \\
0.5 x_{2}^{n+1} \\
0.5 x_{3}^{n+1}
\end{array}\right)\right], \forall n \geqslant 1 .
$$


Choose $\mathbf{x}_{\mathbf{1}}=\left(\begin{array}{c}1 \\ -1 \\ 2\end{array}\right)$ be the initial point. Then, we obtain the numerical results shown in Table 2 and Figures 2 and 3, respectively.

Table 2: Numerical results of Example 5.2.

\begin{tabular}{|c|ccc|c|}
\hline $\mathrm{n}$ & \multicolumn{3}{|c|}{$\mathbf{x}^{\mathrm{n}}=\left(x_{1}^{\mathrm{n}}, x_{2}^{\mathrm{n}}, x_{3}^{\mathrm{n}}\right)^{\top}$} & $\left\|\mathbf{x}_{\mathrm{n}}-\mathrm{p}\right\|$ \\
\hline 1 & $(1.00000000$, & -1.00000000, & $2.00000000)^{\top}$ & 2.44948974 \\
2 & $(0.45843057$, & -0.47840944, & $1.06922917)^{\top}$ & 1.25788918 \\
3 & $(0.23280903$, & -0.23985213, & $0.54051127)^{\top}$ & 0.63551673 \\
4 & $(0.11614084$, & -0.11996289, & $0.27068651)^{\top}$ & 0.31804241 \\
5 & $(0.05808722$, & -0.05997330, & $0.13537353)^{\top}$ & 0.15905004 \\
6 & $(0.02904268$, & -0.02998755, & $0.06768886)^{\top}$ & 0.07952680 \\
7 & $(0.01452138$, & -0.01499369, & $0.03384457)^{\top}$ & 0.03976351 \\
8 & $(0.00726069$, & -0.00749685, & $0.01692230)^{\top}$ & 0.01988176 \\
9 & $(0.00363035$, & -0.00374843, & $0.00846115)^{\top}$ & 0.00994088 \\
10 & $(0.00181517$, & -0.00187421, & $0.00423057)^{\top}$ & 0.00497044 \\
$\vdots$ & $\vdots$ & $\vdots$ & $\vdots$ & $\vdots$ \\
20 & $(1.77263 \mathrm{e}-06$, & $-1.83029 \mathrm{e}-06$, & $4.13142 \mathrm{e}-06)^{\top}$ & $4.85395 \mathrm{e}-06$ \\
\hline
\end{tabular}

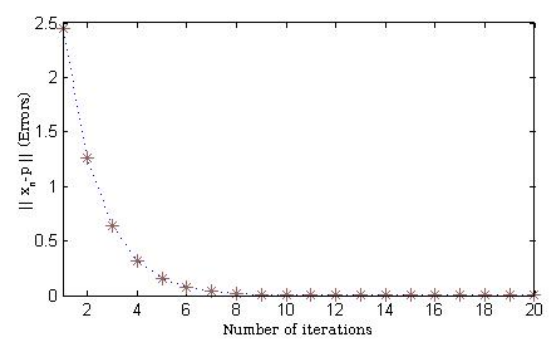

Figure 2: Behavior of convergence error values.

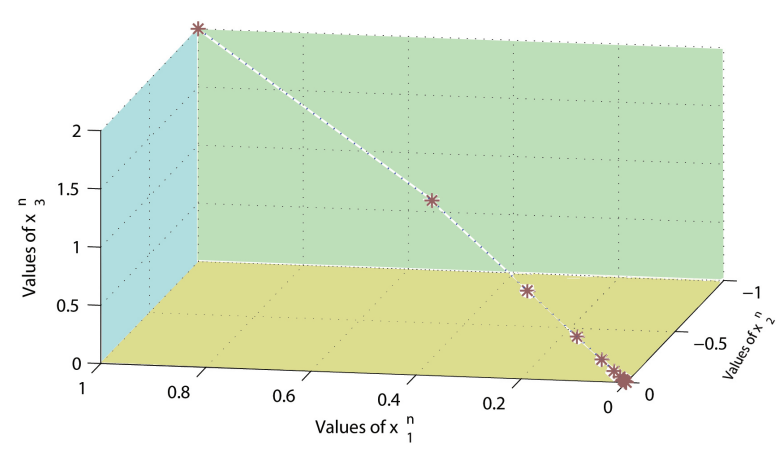

Figure 3: Behavior of convergence values of $\left\{x^{n}\right\}$.

\section{Acknowledgment}

P. Sunthrayuth was supported by RMUTT research foundation scholarship of Rajamangala University of Technology Thanyaburi (Grant No.NRF04066005). This project was supported by the Theoretical and Computational Science (TaCS) Center under ComputationaL and Applied Science for Smart Innovation Cluster (CLASSIC), Faculty of Science, KMUTT.

\section{References}

[1] A. Aleyner, Y. Censor, Best approximation to common Fixed points of a semigroup of nonexpansive operator, J. Nonlinear Convex Anal., 6 (2005), 137-151. 2.9

[2] A. Aleyner, S. Reich, An explicit construction of sunny nonexpansive retractions in Banach spaces, Fixed Point Theory Appl., 2005 (2005), 295-305. 2.9

[3] K. Aoyama, Y. Kimura, W. Takahashi, M. Toyoda, Approximation of common fixed points of a countable family of nonexpansive mapping in a Banach space, Nonlinear Anal., 67 (2007), 2350-2360. 4.1, 4.2, 4.5

[4] H. Attouch, Viscosity solutions of minimization problems, SIAM J. Optim., 6 (1996), 769-806. 1 
[5] W. Auzinger, R. Frank, Asymptotic error expansions for stiff equations: an analysis for the implicit midpoint and trapezoidal rules in the strongly stiff case, Numer. Math., 56 (1989), 469-499. 1

[6] G. Bader, P. Deuflhard, A semi-implicit mid-point rule for stiff systems of ordinary differential equations, Numer. Math., 41 (1983), 373-398. 1

[7] J. Banasiak, L. Arlotti, Perturbations of Positive Semigroups with Applications, Springer-Verlag, London, (2006).

[8] T. D. Benavides, G. L. Acedo, H.-K. Xu, Construction of sunny nonexpansive retractions in Banach spaces, Bull. Austral. Math. Soc., 66 (2002), 9-16. 2.9

[9] F. E. Browder, Convergence theorems for sequences of nonlinear operators in Banach spaces, Math. Z., 100 (1967), $201-225$. 2

[10] S.-S. Chang, Viscosity approximation methods for a finite family of nonexpansive mappings in Banach spaces, J. Math. Anal. Appl., 323 (2006), 1402-1416. 1

[11] R. D. Chen, H. M. He, Viscosity approximation of common fixed points of nonexpansive semigroups in Banach space, Appl. Math. Lett., 20 (2007), 751-757. 1

[12] R. D. Chen, Y. Y. Song, Convergence to common fixed point of nonexpansive semigroup, J. Comput. Anal. Appl., 200 (2007), 566-575. 2.10

[13] P. Cholamjiak, S. Suantai, Viscosity approximation methods for a nonexpansive semigroup in Banach spaces with gauge functions, J. Glob. Optim., 54 (2012), 185-197. 1, 2.11

[14] P. Deuflhard, Recent progress in extrapolation methods for ordinary differential equations, SIAM Rev., 27 (1985), 505-53. 1

[15] K.-J. Engel, R. Nagel, One-Parameter Semigroups for Linear Evolution Equations, Springer-Verlag, New York, (2000). 2

[16] K. Eshita, W. Takahashi, Approximating zero points of accretive operators in general Banach spaces, JP J. Fixed Point Theory Appl., 2 (2007), 105-116. 4.2

[17] Y. F. Ke, C. F. Ma, The generalized viscosity implicit rules of nonexpansive mappings in Hilbert spaces, Fixed Point Theory Appl., 2015 (2015), 21 pages. 1

[18] T.-C. Lim, H.-K. Xu, Fixed point theorems for asymptotically nonexpansive mappings, Nonlinear Anal., 22 (1994), 13451355. 2.4

[19] A. Moudafi, Viscosity approximation methods for fixed point problems, J. Math. Anal. Appl., 241 (2000), 46-55. 1

[20] X. L. Qin, Y. J. Cho, S. M. Kang, Viscosity approximation methods for generalized equilibrium problems and fixed point problems with applications, Nonlinear Anal., 72 (2010), 99-112. 1

[21] C. Schneider, Analysis of the linearly implicit mid-point rule for differential-algebra equations, Electron. Trans. Numer. Anal., 1 (1993), 1-10. 1

[22] S. Somalia, Implicit midpoint rule to the nonlinear degenerate boundary value problems, Int. J. Comput. Math., 79 (2002), 327-332. 1

[23] Y. Song, R. Chen, Viscosity approximative methods to Cesàro means for non-expansive mappings, Appl. Math. Comput., 186 (2007), 1120-1128. 1

[24] Y. S. Song, R. D. Chen, H. Y. Zhou, Viscosity approximation methods for nonexpansive mapping sequences in Banach spaces, Nonlinear Anal., 66 (2007), 1016-1024.

[25] P. Sunthrayuth, Y. J. Cho, P. Kumam, Viscosity Approximation Methods for Zeros of Accretive Operators and Fixed Point Problems in Banach Spaces, Bull. Malays. Math. Sci. Soc., 39 (2016), 773-793. 1

[26] T. Suzuki, Strong convergence of Krasnoselskii and Mann's type sequence for one-parameter nonexpansive semigroup without Bochner integrals, J. Math. Anal. Appl., 305 (2005), 227-239. 2.12

[27] W. Takahashi, Nonlinear Functional Analysis: Fixed point theory and its applications, Yokohama Publishers, Yokohama, (2000). 4.2

[28] M. Van Veldhuxzen, Asymptotic expansions of the global error for the implicit midpoint rule (stiff case), Computing, 33 (1984), 185-192. 1

[29] H.-K. Xu, Another control condition in an iterative method for nonexpansive mappings, Bull. Austral. Math. Soc., 65 (2002), 109-113. 2.13

[30] H.-K. Xu, Viscosity approximation methods for nonexpansive mappings, J. Math. Anal. Appl., 298 (2004), 279-291. 1

[31] H.-K. Xu, M. A. Alghamdi, N. Shahzad, The viscosity technique for the implicit midpoint rule of nonexpansive mappings in Hilbert spaces, Fixed Point Theory Appl., 2015 (2015), 12 pages. 1

[32] C. Schneider, Analysis of the linearly implicit mid-point rule for differential-algebra equations, Electron. Trans. Numer. Anal., 1 (1993), 1-10. 1 